\title{
Injection orbit matching for a rapid cycling synchrotron
}

\author{
Xiaohan Lu, ${ }^{1,2,3}$ Ming-Yang Huang, ${ }^{1,3}$ and Sheng Wang ${ }^{1,3, *}$ \\ ${ }^{1}$ Institute of High Energy Physics, Chinese Academy of Sciences, Beijing 100049, China \\ ${ }^{2}$ University of Chinese Academy of Sciences, Beijing 100049, China \\ ${ }^{3}$ Dongguan Neutron Science Center, Dongguan 523808, China
}

(Received 14 February 2018; published 28 June 2018)

\begin{abstract}
The rapid cycling synchrotron (RCS) of the China Spallation Neutron Source (CSNS) employs painting injection to achieve a uniform beam distribution and to suppress the space charge effect. In the painting injection, a mismatch between the injection and the circulating beam orbit can cause nonuniform beam distribution and larger emittance, which may lead to beam loss. To match the injection beam orbit and the circulating beam orbit, it is necessary to identify the injection beam orbit relative to the circulating beam orbit at the injection point. However, the relative injection beam orbit is hard to measure directly. Theoretically, the relative injection beam orbit can be deduced from the turn-by-turn beam position of a single-turn injected beam, but in the RCS of CSNS, the intensity of a single-turn injected beam is too low to measure with a sufficient signal-noise ratio using a beam position monitor. In this paper, two effective methods based on multiturn injection and turn-by-turn beam position data are given to perform the match between the injection and the circulating beam orbit. Simulation results and practical application during the beam commissioning of the RCS of CSNS show the validity of the methods.
\end{abstract}

DOI: 10.1103/PhysRevAccelBeams.21.062802

\section{INTRODUCTION}

The China Spallation Neutron Source (CSNS) is a highintensity, proton accelerator-based, $100 \mathrm{~kW}$ pulsed neutron source [1]. Its accelerators consist of an $80 \mathrm{MeV} \mathrm{H}$ linac and a $1.6 \mathrm{GeV}$ proton rapid cycling synchrotron (RCS) with a repetition rate of $25 \mathrm{~Hz}$ [2]. The schematic layout of the accelerator is shown in Fig. 1, and its main parameters are shown in Table I. The layout of the injection system is shown in Fig. 2. Eight painting bump magnets (BH1-BH4 for horizontal and BV1-BV4 for vertical) and four chicane bump magnets (BC1-BC4) are accommodated in an uninterrupted straight section [3,4]. Painting injection $[5,6]$ is employed in the RCS to achieve a uniform beam distribution and to suppress the space charge effect.

To control the painting process more precisely, it is necessary to identify the injection beam orbit relative to the circulating beam orbit at the injection point, which is $\left(\Delta x / \Delta y, \Delta x^{\prime} / \Delta y^{\prime}\right)$ in the transverse phase space. Hereafter, we choose $\left(\Delta x, \Delta x^{\prime}\right)$ as the object of study. If the relative injection beam orbit is identified, the injection and the circulating beam orbit can be well matched. A mismatch between the injection and the circulating beam orbit can

\footnotetext{
*wangs@ihep.ac.cn
}

Published by the American Physical Society under the terms of the Creative Commons Attribution 4.0 International license. Further distribution of this work must maintain attribution to the author(s) and the published article's title, journal citation, and DOI. lead to nonuniform beam distribution and larger emittance, which may lead to additional beam loss. Taking the RCS of CSNS as an example, an anticorrelated painting process was simulated by using the particle-tracking code ORBIT [7]. Simulations show the effects of the injection orbit mismatch on the painting emittance and the beam loss.

In anticorrelated painting, horizontal painting fills the RCS acceptance from the center to the outer phase space, and the matching conditions are $\Delta x=0, \Delta x^{\prime}=0$. In the simulation, we set $\Delta x^{\prime}=0$ and scan $\Delta x$ from 0 to $10 \mathrm{~mm}$. There are 200 turns in the injection painting process, and the next 2000 turns for acceleration were considered in the simulation.

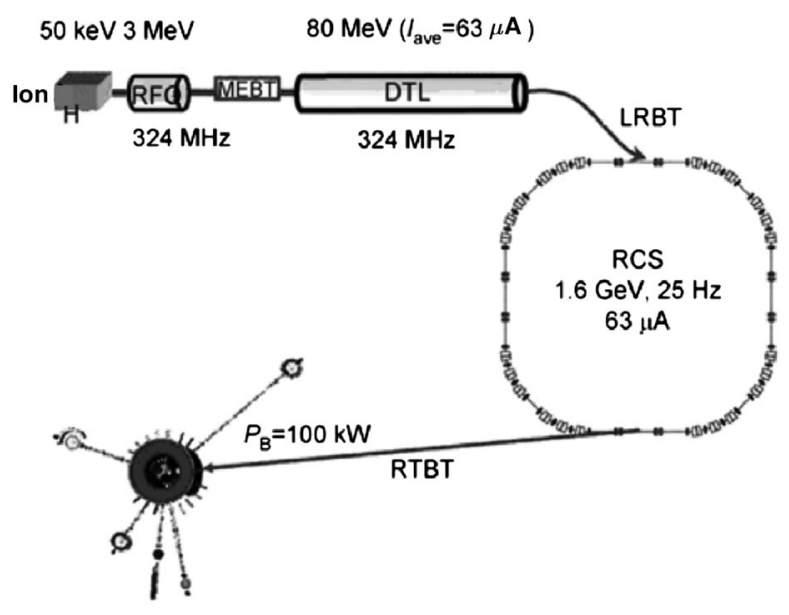

FIG. 1. The schematic layout of CSNS. 
TABLE I. RCS design parameters.

\begin{tabular}{lc}
\hline \hline Parameters & Value \\
\hline Circumference & $227.92 \mathrm{~m}$ \\
Superperiodicity & 4 \\
Injection energy & $80 \mathrm{MeV}$ \\
Single injection beam peak current & $15 \mathrm{~mA}$ \\
Extraction energy & $1.6 \mathrm{GeV}$ \\
Repetition rate & $25 \mathrm{~Hz}$ \\
Extraction beam power & $100 \mathrm{~kW}$ \\
Design betatron tunes $(\mathrm{h} / \mathrm{v})$ & $4.86 / 4.78$ \\
\hline \hline
\end{tabular}

As shown in Fig. 3, the emittance increases with the increase of $\Delta x$ (mismatch), and the beam loss is significantly increased when $\Delta x>5 \mathrm{~mm}$.

The identification of the relative injection beam orbit is critical to perform good injection matching. However, since the injection beam is part of the transport line and the circulating beam is part of the synchrotron, the relative injection beam orbit is hard to measure directly. Theoretically, the relative injection beam orbit can be calculated by using the transfer matrix with the turn-byturn beam position of a single-turn injected beam. For CSNS, however, the maximum intensity of a single-turn injected beam is $15 \mathrm{~mA}$, which is too low to measure with a

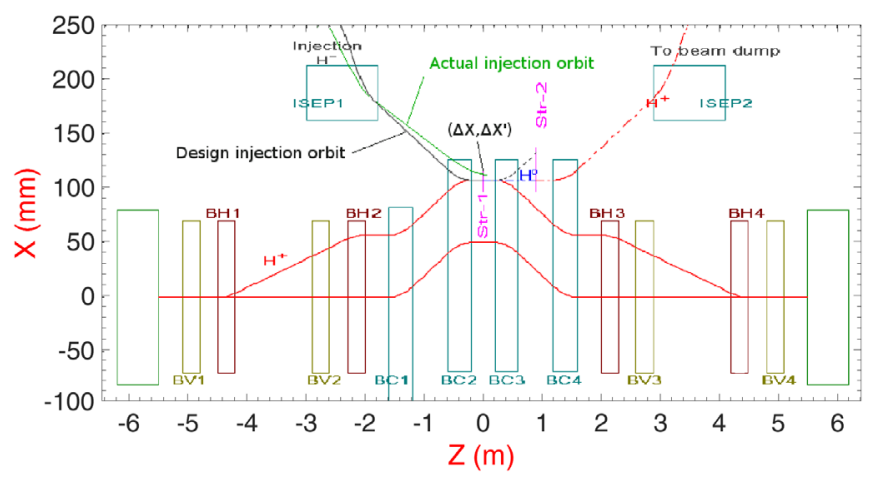

FIG. 2. Schematic layout of the injection system.

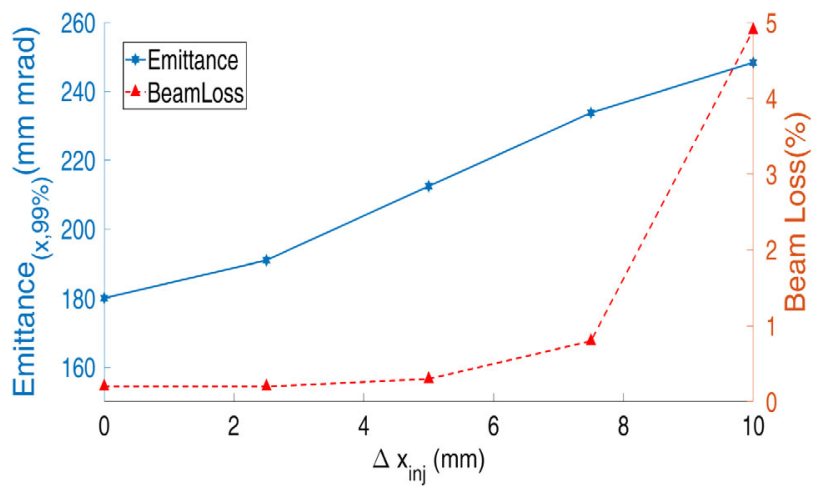

FIG. 3. Effects of the injection orbit mismatch on emittance (blue solid line) and beam loss (red dashed line). sufficient signal-noise ratio using a beam position monitor (BPM). So, the beam current should be increased with multiturn injection to gain a larger signal-noise ratio for the BPM, while the identification of the phase space coordinates becomes more complicated.

In this paper, two methods based on multiturn injection and turn-by-turn beam position data are introduced to identify the injection orbit relative to the circulating orbit. Simulation results and practical application during the beam commissioning of the RCS of CSNS show the validity of the methods.

\section{METHOD TO IDENTIFY THE INJECTION ORBIT BASED ON TRANSFER MATRIX}

\section{A. Basic principle}

The matrix of betatron oscillation can be written as

$$
\left[\begin{array}{c}
\Delta x_{i} \\
\Delta x_{i}^{\prime}
\end{array}\right]=M_{i}\left[\begin{array}{c}
\Delta x_{i n j} \\
\Delta x_{i n j}^{\prime}
\end{array}\right]
$$

where $\Delta x_{i n j}, \Delta x_{i n j}^{\prime}$ and $\Delta x_{i}, \Delta x_{i}^{\prime}$ are the transverse phase space coordinates relative to the circulating orbit at the injection point and the $i$ th BPM, respectively, and $M_{i}$ is the transfer matrix from the injection point to the $i$ th BPM.

As a BPM detects the charge center of a beam, for an injected beam of $m$ turns, the transverse phase space coordinates at the $j$ th BPM after $n$ turns can be derived as

$$
\left[\begin{array}{c}
\Delta x_{j} \\
\Delta x_{j}^{\prime}
\end{array}\right]=M_{i n j-j}\left(M^{n-1}+M^{n-2}+\cdots+M^{n-m}\right) / m\left[\begin{array}{c}
\Delta x_{i n j} \\
\Delta x_{i n j}^{\prime}
\end{array}\right],
$$

where $\Delta x_{j}$ and $\Delta x_{j}^{\prime}$ are the phase space coordinates of the circulating beam, $\Delta x_{i n j}$ and $\Delta x_{i n j}^{\prime}$ are the injection beam coordinates at the injection point, $M_{i n j-j}$ is the transfer matrix from injection point to the $j$ th BPM, and $M$ is the one-turn transfer matrix.

In Eq. (2), $\Delta x_{j}$ can be measured directly by using a turnby-turn BPM, and $\Delta x_{j}^{\prime}$ can be obtained with a pair of BPMs located in a drift space [8]. Here a more general algorithm is introduced to obtain $\Delta x_{i n j}$ and $\Delta x_{i n j}^{\prime}$ :

$$
\begin{gathered}
m\left[\begin{array}{l}
\Delta x_{1} \\
\Delta x_{1}^{\prime}
\end{array}\right]=M_{1}(n)\left[\begin{array}{l}
\Delta x_{i n j} \\
\Delta x_{i n j}^{\prime}
\end{array}\right], \\
m\left[\begin{array}{l}
\Delta x_{2} \\
\Delta x_{2}^{\prime}
\end{array}\right]=M_{2}(n)\left[\begin{array}{l}
\Delta x_{i n j} \\
\Delta x_{i n j}^{\prime}
\end{array}\right], \\
\Rightarrow \quad \\
m\left[\begin{array}{l}
\Delta x_{1} \\
\Delta x_{2}
\end{array}\right]=\left[\begin{array}{ll}
{\left[M_{1}(n)\right]_{11}} & {\left[M_{1}(n)\right]_{12}} \\
{\left[M_{2}(n)\right]_{11}} & {\left[M_{2}(n)\right]_{12}}
\end{array}\right]\left[\begin{array}{l}
\Delta x_{i n j} \\
\Delta x_{i n j}^{\prime}
\end{array}\right],
\end{gathered}
$$


where $m$ is the injection turn, $n$ is the circulating turn starting from the beginning of injection, and $M_{j}(n)=$ $M_{i n j-j}\left(M^{n-1}+M^{n-2}+\cdots+M^{n-m}\right)$ is the transfer matrix from the injection point to the $j$ th $(j=1,2)$ BPM.

\section{B. Numerical simulations}

In the simulation and beam commissioning, the primary errors which should be considered are the errors of the transfer matrix and the BPM resolution. In the beam commissioning, the tune deviation due to the errors of the transfer matrix can be controlled to less than 0.005 by making optics correction with LOCO (linear optics from closed orbits) [9]. To estimate the error effects of the transfer matrix, a simulation was done by using Accelerator ToolBox(AT), which is a collection of tools to model particle accelerators in the MATLAB environment [10]. When the tune deviation due to the errors of a transfer matrix is less than 0.005 , the errors of the reconstructed $\Delta x_{i n j}$ and $\Delta x_{i n j}^{\prime}$ in Eq. (5) are less than $\pm 0.5 \mathrm{~mm}$ and $\pm 0.03 \mathrm{mrad}$, so only the BPM errors are considered in the following simulations.

In the simulation, the random BPM errors, normally distributed, were generated with different BPM resolutions: $0.5,1.5$, and $2.5 \mathrm{~mm}$, respectively. Three turns of beam were injected, the circulating turn [ $n$ in Eq. (2)] was 3, and the preset horizontal phase space coordinates at the injection point were ( $5 \mathrm{~mm}, 1 \mathrm{mrad})$. R4BPM12 and R4BPM02 were marked as the observation points during the tracking. A total of 200 sets of BPM errors were simulated, and the reconstructed phase space coordinates at the injection point were obtained by using Eq. (5). The results are shown in the histograms in Fig. 4, in which the left-hand plots are the position of the phase space coordinates and the right-hand plots are the angular coordinates. The mean values $(\mu)$ and the standard deviation $(\delta)$ of the results were obtained by fitting the distribution to a Gaussian function. The simulated means of $\left(\Delta x_{i n j}, \Delta x_{i n j}^{\prime}\right)$ with different BPM resolutions are close to the preset values of $(5 \mathrm{~mm}, 1 \mathrm{mrad})$, reflecting the validity of the method. The standard deviations of $\left(\Delta x_{i n j}, \Delta x_{i n j}^{\prime}\right)$ are $(1.65 \mathrm{~mm}, 0.457 \mathrm{mrad}),(4.75 \mathrm{~mm}, 1.4 \mathrm{mrad})$, and $(9.13 \mathrm{~mm}$, $2.68 \mathrm{mrad}$ ) for a BPM resolution of $0.5,1.5$, and $2.5 \mathrm{~mm}$, respectively. The simulation result is very sensitive to the resolution of the BPM, and a high BPM resolution is required in this algorithm. For the RCS of CSNS, the nominal BPM resolution is about $2 \mathrm{~mm}$, and it is difficult to achieve a highaccuracy result using this algorithm. To improve the accuracy, an improved algorithm is proposed in Sec. III.

\section{METHOD TO IDENTIFY THE INJECTION ORBIT BASED ON FOURIER TRANSFORM}

\section{A. Basic principle}

When the injected beam deviates from the closed orbit of circulating beam at the injection point, the betatron oscillation can be detected by a BPM. Theoretically, the
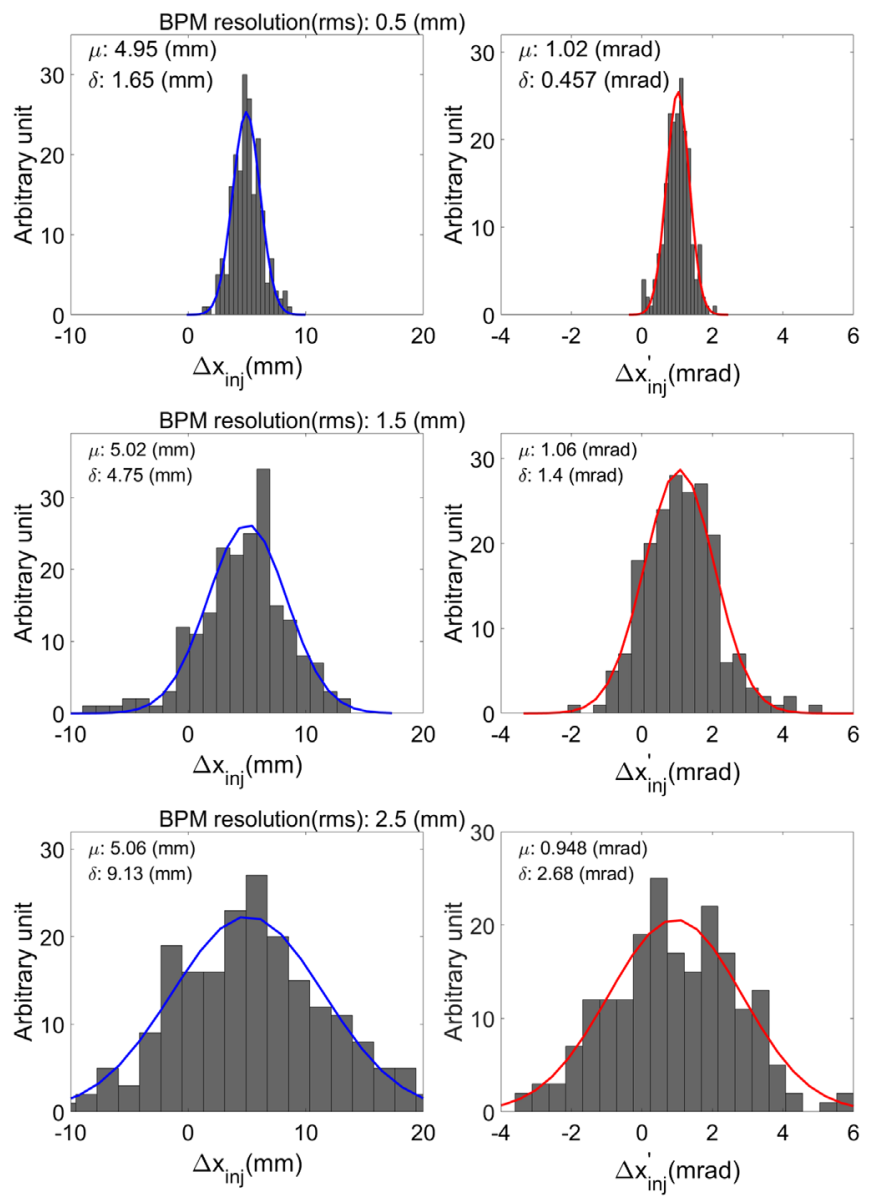

FIG. 4. Simulation results of reconstructing the phase space coordinates relative to the circulating orbit at the injection point, obtained by repeating calculations 200 times with the algorithm described in Sec. II A. In the simulations, the injection turn [ $m$ in Eq. (2)] is 3, and the circulating turn [ $n$ in Eq. (2)] is also 3. The left-hand plots are the position of the phase space coordinates, and the right-hand plots are the angular coordinates. The mean value $(\mu)$ and the width $(\delta)$ were obtained by fitting the distribution to a Gaussian function. The simulation results with a BPM resolution of $0.5,1.5$, and $2.5 \mathrm{~mm}$ are given.

displacement from the reference orbit after $n$ turns at a specified BPM can be written as [11]

$$
\begin{aligned}
\Delta x(n)= & \Delta x_{0} \cos \left(2 \pi n v_{x}\right) \\
& +\left(\Delta x_{0} \alpha_{x}+\Delta x_{0}^{\prime} \beta_{x}\right) \sin \left(2 \pi n v_{x}\right),
\end{aligned}
$$

where $\Delta x_{0}$ and $\Delta x_{0}^{\prime}$ are the initial phase space coordinates at the specified BPM, $v_{x}$ is the betatron tune, $n$ is the turn number, and $\alpha_{x}$ and $\beta_{x}$ are the Twiss parameters. Generally, the above oscillation is a simple harmonic oscillation with respect to turn number $n$. Let

$$
\begin{aligned}
& a_{1}=\Delta x_{0}, \\
& b_{1}=\Delta x_{0} \alpha_{x}+\Delta x_{0}^{\prime} \beta_{x} .
\end{aligned}
$$


Then Eq. (6) can be expressed as

$$
\begin{aligned}
\Delta x(n) & =a_{1} \cos \left(2 \pi n v_{x}\right)+b_{1} \sin \left(2 \pi n v_{x}\right) \\
& =c_{1} \cos \left(2 \pi n v_{x}+\phi_{1}\right),
\end{aligned}
$$

where $c_{1}=\sqrt{a_{1}^{2}+b_{1}^{2}}$ and $\phi_{1}=\arctan \frac{-b_{1}}{a_{1}}$.

The Fourier series of Eq. (9) can be written as

$$
\begin{aligned}
\Delta x(n) & =\frac{c_{1}}{2} e^{-j \phi_{1}} e^{-j 2 \pi n v_{x}}+\frac{c_{1}}{2} e^{j \phi_{1}} e^{j 2 \pi n v_{x}} \\
& =X_{-1} e^{-j 2 \pi n v_{x}}+X_{1} e^{j 2 \pi n v_{x}},
\end{aligned}
$$

where $X_{-1}=\frac{c_{1}}{2} e^{-j \phi_{1}}$ and $X_{1}=\frac{c_{1}}{2} e^{j \phi_{1}}$ are Fourier series coefficients. Since $\Delta x(n)$ is a periodic signal, its Fourier transform can be computed directly as [12]

$$
\begin{aligned}
X(v) & =X_{-1} \delta\left(v+v_{x}\right)+X_{1} \delta\left(v-v_{x}\right) \\
& =\frac{c_{1}}{2} e^{-j \phi_{1}} \delta\left(v+v_{x}\right)+\frac{c_{1}}{2} e^{j \phi_{1}} \delta\left(v-v_{x}\right),
\end{aligned}
$$

where $\delta$ is the delta function. According to Eq. (11), the real part $\operatorname{Re}\left[X\left(v_{x}\right)\right]$ and imaginary part $\operatorname{Im}\left[X\left(v_{x}\right)\right]$ can be obtained, respectively, as

$$
\begin{aligned}
& \operatorname{Re}\left[X\left(v_{x}\right)\right]=\operatorname{Re}\left[\frac{c_{1}}{2} e^{j \phi_{1}}\right]=\frac{c_{1}}{2} \cos \phi_{1}=\frac{a_{1}}{2}, \\
& \operatorname{Im}\left[X\left(v_{x}\right)\right]=\operatorname{Im}\left[\frac{c_{1}}{2} e^{j \phi_{1}}\right]=\frac{c_{1}}{2} \sin \phi_{1}=-\frac{b_{1}}{2} .
\end{aligned}
$$

Based on Eqs. (7), (8), (12), and (13), a mapping matrix can be set up as

$$
\left[\begin{array}{l}
\operatorname{Re}\left[X\left(v_{x}\right)\right] \\
\operatorname{Im}\left[X\left(v_{x}\right)\right]
\end{array}\right]=R\left(v_{x}\right)\left[\begin{array}{l}
\Delta x_{0} \\
\Delta x_{0}^{\prime}
\end{array}\right]
$$

where

$$
R\left(v_{x}\right)=\left[\begin{array}{cc}
\frac{1}{2} & 0 \\
-\frac{\alpha_{x}}{2} & -\frac{\beta_{x}}{2}
\end{array}\right] .
$$

According to Eq. (2), with $m$ turns of injected beam, the transfer matrix from the injection point to a specified BPM after $k$ turns of transfer is

$$
\left[\begin{array}{c}
\Delta x_{0} \\
\Delta x_{0}^{\prime}
\end{array}\right]=M_{m / k}\left[\begin{array}{c}
\Delta x_{i n j} \\
\Delta x_{i n j}^{\prime}
\end{array}\right],
$$

where

$M_{m / k}=M_{i n j-j}\left(M^{k-1}+M^{k-2}+\cdots+M^{k-m}\right) / m$.

The real and imaginary parts of $X\left(v_{x}\right)$ can be described by the matrix $A=R\left(v_{x}\right) M_{m / k}$ and the initial phase space coordinates at the injection point:

$$
\left[\begin{array}{l}
\operatorname{Re}\left[X\left(v_{x}\right)\right] \\
\operatorname{Im}\left[X\left(v_{x}\right)\right]
\end{array}\right]=A\left[\begin{array}{c}
\Delta x_{i n j} \\
\Delta x_{i n j}^{\prime}
\end{array}\right]
$$

where $X\left(v_{x}\right)$ can be obtained from the turn-by-turn data through Fourier analysis and the matrix $A$ can be obtained by a beam-based measurement.

\section{B. Numerical simulations}

The simulation was performed in a similar way to what was done in Sec. II B. Most of the simulation conditions were consistent with those of Sec. II B, except that the total number of tracking turns was increased to 20. In the simulation, $m$ and $k$ in Eq. (17) were both 3, and there were 18 circulating turns after injection [ $n$ in Eq. (6)]. The simulation results are shown in Fig. 5. For the different BPM resolutions, the mean values of $\left(\Delta x_{i n j}, \Delta x_{i n j}^{\prime}\right)$ are consistent with each other and close to the preset values of ( $5 \mathrm{~mm}, 1 \mathrm{mrad}$ ), indicating the validity of the algorithm. The standard deviations of $\left(\Delta x_{i n j}, \Delta x_{i n j}^{\prime}\right)$ were $(0.214 \mathrm{~mm}$, $0.0452 \mathrm{mrad}),(0.817 \mathrm{~mm}, 0.139 \mathrm{mrad})$, and $(1.41 \mathrm{~mm}$, $0.223 \mathrm{mrad}$ ) for a BPM resolution of $0.5,1.5$, and $2.5 \mathrm{~mm}$, respectively, which is quite small even with the large BPM resolution of $2.5 \mathrm{~mm}$. The simulation shows the good accuracy of the algorithm.
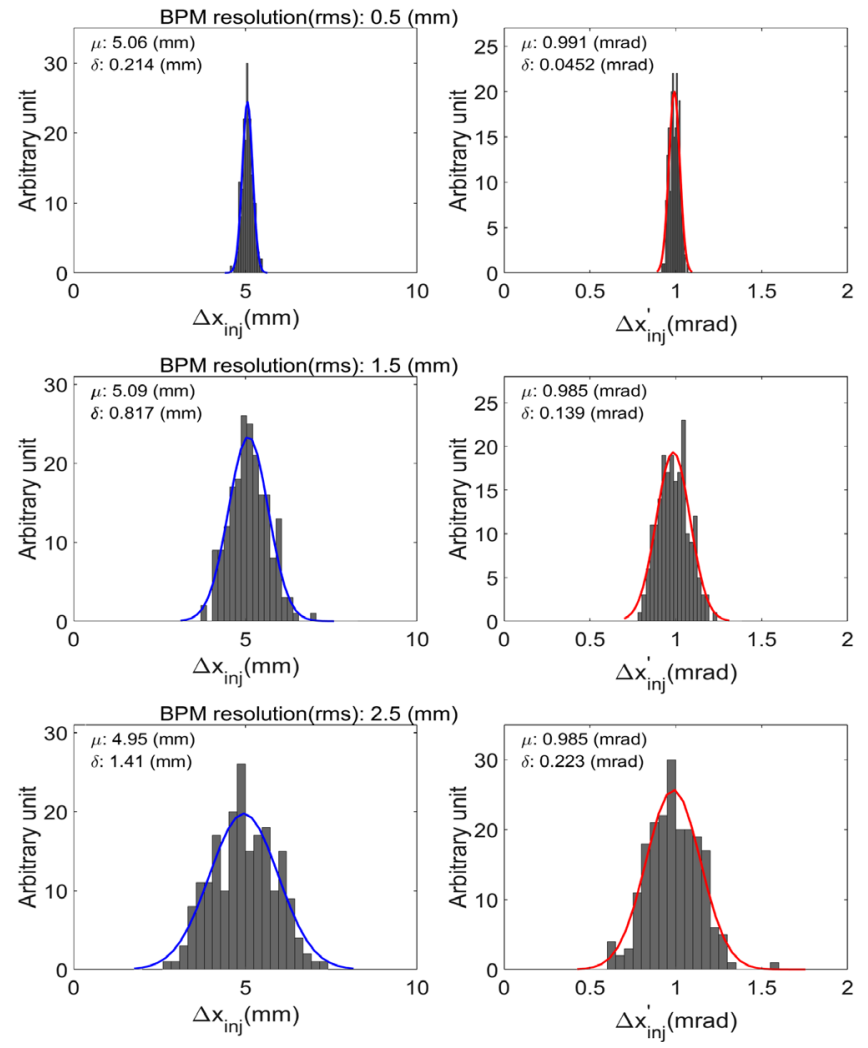

FIG. 5. Similar to Fig. 4 but with the total tracking turns increased to 20. The $m$ and $k$ in Eq. (17) were both 3, and the number of circulation turns after injection [ $n$ in Eq. (6)] was 18. R4BPM12 was marked as the observation point during tracking. 

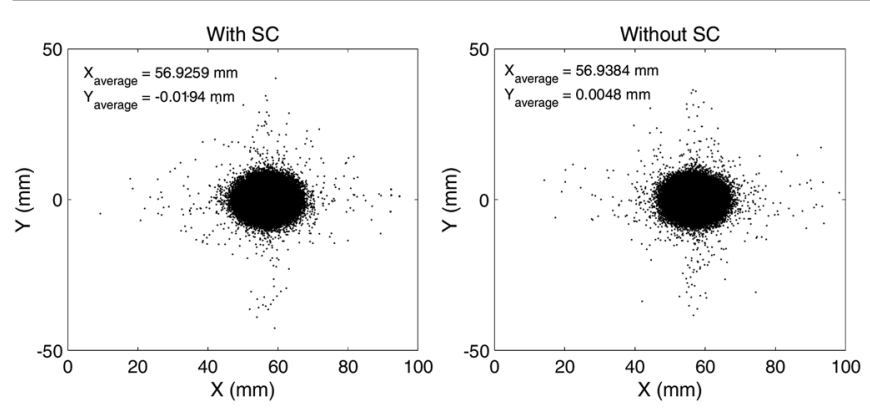

FIG. 6. Comparison of the tracking results with and without space charge. Left: The tracking result with the space charge effect. Right: The case without the space charge effect.

The above analysis and simulations are based on the linear condition. As mentioned in Sec. II A, the BPM detects the charge center of a beam, so under the linear condition the beam position of a multiturn injected beam is equal to the average of the beam positions of each single pulse. However, in practice, a few factors could break the linear condition, in which the space charge is one of the main nonlinear factors. A simulation was done to evaluate the effect of space charge on the beam position with multiturn injection.

The processes of injection with and without a space charge effect were simulated by using ORBIT. The number of turns of injected beam was 3, each turn of the injected beam contained 40000 macroparticles, which corresponds to a peak current of $10 \mathrm{~mA}$, and the first 20 turns were tracked. The simulation results are shown in Fig. 6, in which the left-hand plot shows the tracking result in real space with the space charge effect, while the right-hand plot shows the case without the space charge effect. It can be seen that for the three-turn injected beam with a peak current of $10 \mathrm{~mA}$, after 20 turns, the effect of the space charge on the beam distribution as well as the beam position is small enough to be ignored.

\section{MACHINE STUDY}

\section{A. Measurement and calculation}

The method from Sec. III was tested and applied in the beam commissioning of the RCS of CSNS. The beam commissioning was still in the early stage, and the closed orbit distortion (COD) had not been well corrected, so the machine study was conducted only for this method.

The machine study was performed in the dc mode of the RCS, in which the beam was injected and extracted with the energy of $80 \mathrm{MeV}$ without acceleration. The harmonic number of the RCS is 2, but in the machine study, the chopped beam was injected into only one of the two buckets, and three pulses from the linac were injected into the RCS with a peak current of $12 \mathrm{~mA}$. Two rf cavities were utilized to provide a rf voltage of $24 \mathrm{kV}$, and the synchrotron period was about $0.36 \mathrm{~ms}$. After the injection, the first 18 turns of BPM data were used in the calculation. The circulating period is about $1.96 \mu \mathrm{s}$, and 18 turns circulating takes about $0.035 \mathrm{~ms}$, which is much less than the period of synchrotron oscillation, so the effect of synchrotron oscillation on the transverse position of the beam is negligible. The chromaticity was corrected to -0.5 by using two families of sextupoles, and the rms momentum spread of the injected beam was about $0.1 \%$, so the damping of the betatron oscillations [11] in the first 18 turns could be ignored. BPMs were selected in the dispersion-free area to avoid the influence of the dispersion. The matrix $A$ was obtained from a beam-calibrated model. The measured tune in the horizontal direction was 4.822, while the tune calculated with the calibrated model was 4.824 , and, according to the simulation result in Sec. II B, the errors of the transfer matrix could be ignored.

Two BPMs were selected as the observation points. Figure 7 shows the measurement results, in which the betatron oscillation can be clearly observed.

The phase space coordinates of the injected beam at the injection point were reconstructed with turn-by-turn data from different BPMs, as listed in Table II. Considering the resolution of the BPM, the consistency of the results obtained from two different BPMs is satisfactory.

The relative position and angle of the injected beam can be corrected by using the chicane bump in the RCS and dipole correctors in the beam transport line. When the deviations of the injected beam are corrected, the betatron oscillation can be suppressed. Figure 8 shows the detected betatron oscillation before and after the correction.

Since the method is based on the turn-by-turn BPM data, the accuracy of the result depends on the resolution of the BPMs. However, in practice, the stability of the injection beam and the fluctuations of the bump magnets are also error sources. In the machine study, it is difficult to evaluate the effect of these factors individually. However, the overall influence of these errors can be extracted by repeated
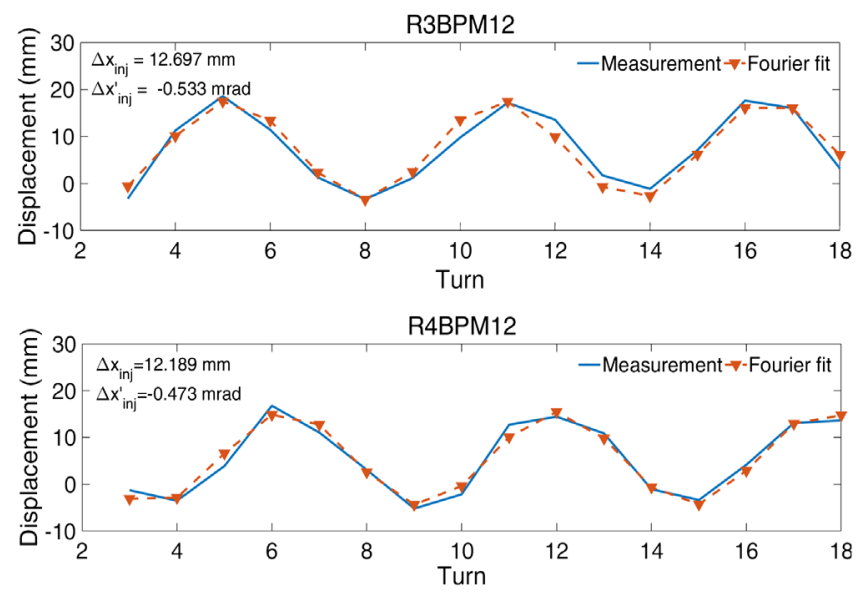

FIG. 7. The measurement results of betatron oscillation at two different BPMs (blue solid lines) and their Fourier fit (red dashed lines with triangles). 
TABLE II. Results of the reconstructed phase space coordinates at the injection point, based on the measured data.

\begin{tabular}{lc}
\hline \hline BPM & Injection $\left(\Delta x_{i n j}, \Delta x_{i n j}^{\prime}\right)(\mathrm{mm}, \mathrm{mrad})$ \\
\hline R3BPM12 & $(12.697,-0.533)$ \\
R4BPM12 & $(12.189,-0.473)$ \\
\hline \hline
\end{tabular}

measurement. Figure 9 shows the statistics results of 11 beam tests, with the turn-by-turn data obtained from four BPMs. The left-hand plot is the position of the phase space coordinates, and the right-hand plot is the angle. The mean value of $\left(\Delta x_{i n j}, \Delta x_{i n j}^{\prime}\right)$ is $(13.8 \mathrm{~mm},-0.486 \mathrm{mrad})$. The standard deviation of $\left(\Delta x_{i n j}, \Delta x_{i n j}^{\prime}\right)$ is $(1.77 \mathrm{~mm}$, $0.285 \mathrm{mrad}$ ), and this can be used to estimate the effects of the overall errors.

\section{B. Error analysis}

Besides the BPM resolution, there are other error sources in the measurement. In the simulation, only the BPM resolution was considered. To evaluate the error effect, further simulation studies were done, in which the overall errors were equivalent to the resolution of BPMs. It is found that, when the BPM resolution is set to $3.5 \mathrm{~mm}$, the standard deviations obtained in the simulation are very close to those in the measurement, as shown in Fig. 10.

To investigate the level of injection deviation $\left(\Delta x_{i n j}, \Delta x_{i n j}^{\prime}\right)$ which generates the residual betatron oscillation in Fig. 8, the simulations were repeated with a BPM resolution of $3.5 \mathrm{~mm}$.

In the simulation, the injection deviations were set to (1 $\mathrm{mm},-0.1 \mathrm{mrad}),(2 \mathrm{~mm},-0.1 \mathrm{mrad})$, and $(1 \mathrm{~mm}$, $-0.2 \mathrm{mrad})$, respectively. The number of injection turn was 3 , and the first 18 turns were tracked. To compare with the residual betatron oscillation, an additional $5 \mathrm{~mm}$ COD was
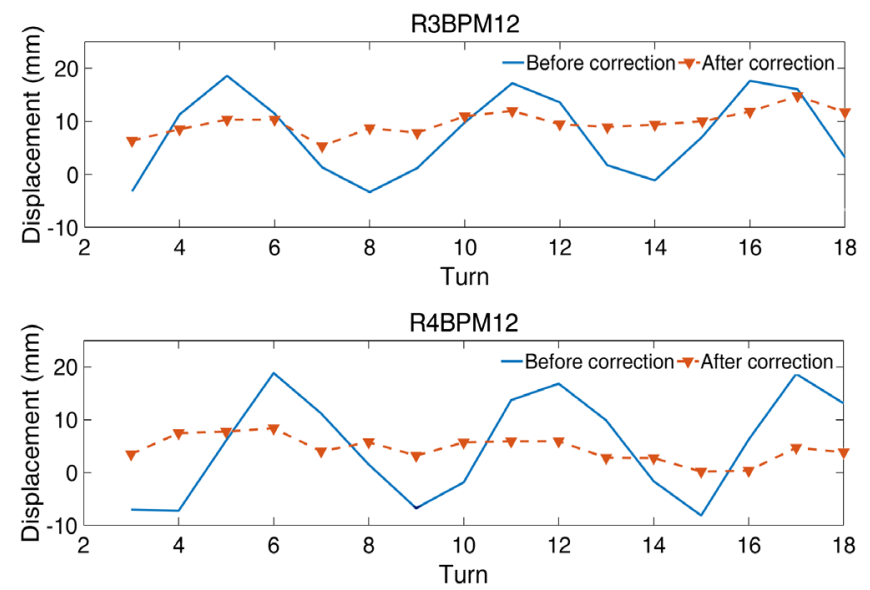

FIG. 8. The detected betatron oscillation at two different BPMs before (blue solid lines) and after (red dashed lines with triangles) the correction for the injection beam.
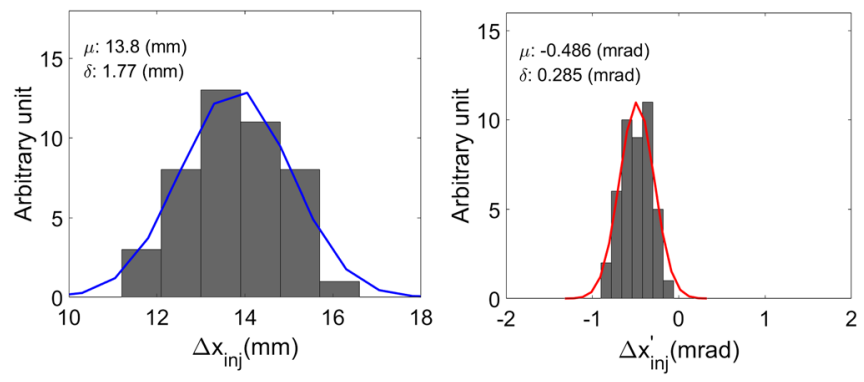

FIG. 9. The statistics of 11 beam tests in the machine study.

added to the simulation results. R4BPM12 was marked as the observation point.

The simulation results compared with the residual betatron oscillation are shown in Fig. 11. The red dashed line is the residual betatron oscillation, which is the same as for the second plot in Fig. 8, and the other lines are three repeated simulation results with a BPM resolution of $3.5 \mathrm{~mm}$. It can be found that the simulation results with an injection deviation of $(1 \mathrm{~mm},-0.1 \mathrm{mrad})$ are close to the residual oscillation in the measurement. So we estimate that the residual betatron oscillation in the measurement corresponds to an injection deviation around ( $1 \mathrm{~mm},-0.1 \mathrm{mrad})$.

In the machine study, due to the limited BPM resolution and the other error effects, it is difficult to further identify such a small injection deviation. The residual matching error is acceptable in the beam commissioning of CSNS.

\section{DISCUSSION}

The first method is very sensitive to the BPM errors, and the accuracy of the result depends on both the BPM resolution and offset. The reason is that the errors are involved in the calculation directly, and there is no subprocess to suppress the effects of the BPM errors. The second method is more robust and is relatively insensitive to the errors. The simulation and machine study show that the standard deviation of the results is quite small even with a relative low BPM resolution. The key point is that the Fourier curve fitting is utilized to calculate the real and imaginary parts of oscillation data, and the influence of the BPM errors is suppressed during the fitting.
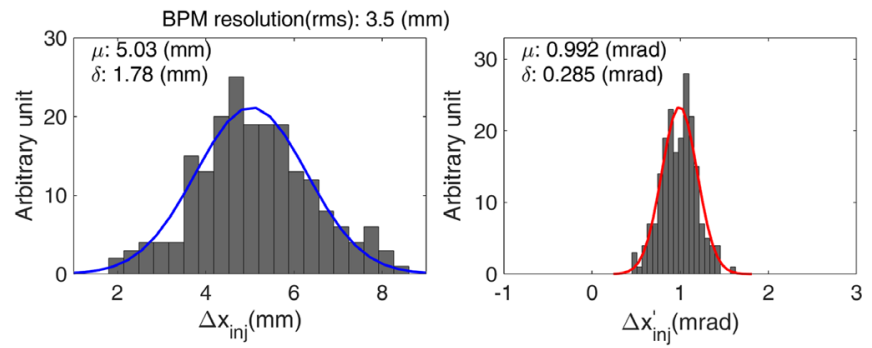

FIG. 10. Similar to Fig. 5 but with the BPM resolution set to $3.5 \mathrm{~mm}$. 

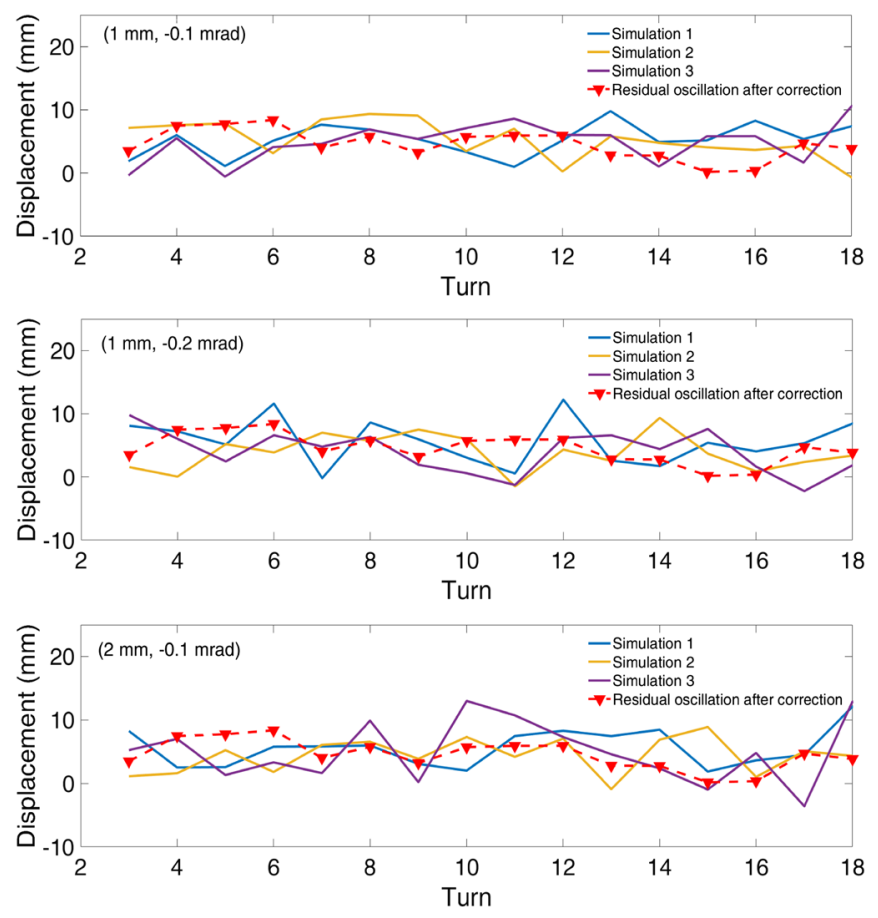

FIG. 11. Three repeated simulation results (shown as simulations 1, 2, and 3) compared with the residual betatron oscillation (red dashed line with triangles) after a correction in the second plot in Fig. 8. Three turns of beam were injected, R4BPM12 was marked as the observation point, the BPM resolution was set to $3.5 \mathrm{~mm}$, and the injection deviations were set to $(1 \mathrm{~mm},-0.1 \mathrm{mrad}),(1 \mathrm{~mm},-0.2 \mathrm{mrad})$, and $(2 \mathrm{~mm}$, $-0.1 \mathrm{mrad})$, respectively.

Compared with the method based on the single-pass mode and BPM pairs, the present two methods based on multiturn injection are more universal. Any BPM can be marked as an observation point in the calculation, and the accumulation of beam intensity can enhance the signalnoise ratio of the BPM.

\section{CONCLUSION}

In painting injection, the mismatch between the injection and the circulating beam orbit will result in a large painting emittance and nonuniformity of the beam distribution, which may lead to beam loss. Because of the special location and limitation of the detector, the identification of the phase space coordinates of the injection beam at the injection point is difficult. Two effective methods were introduced to identify the phase space coordinates at the injection point, based on multiturn injection and turn-by-turn BPM data, and both methods were investigated by simulation studies. Because of the limited BPM resolution, only the second method was tested in the beam commissioning of the RCS of CSNS, and the results can be utilized in the injection orbit matching. The detected betatron oscillation was clearly suppressed after the correction, indicating that the injection and the circulating beam orbit were well matched.

\section{ACKNOWLEDGMENTS}

The authors thank T. G. Xu, S. Y. Xu, J. L. Sun, P. Zhu, and other CSNS colleagues for the discussion and consultations.

[1] S. Wang, S. X. Fang, Q. Qin, J. Y. Tang, and J. Wei, Introduction to the overall physics design of CSNS accelerators, Chin. Phys. C 33, 1 (2009).

[2] J. Wei, S. N. Fu, J. Y. Tang, J. Z. Tao, D. S. Wang, F. W. Wang, and S. Wang, China Spallation Neutron SourceAn overview of application prospects, Chin. Phys. C 33, 1033 (2009).

[3] J. Y. Tang, J. Qiu, S. Wang, and J. Wei, Physics design and study of the BSNS RCS injection system, Chin. Phys. C 30, 1184 (2006).

[4] M. Y. Huang, S. Wang, J. Qiu, N, Wang, and S. Y. Xu, The effects of injection beam parameters and foil for CSNS/ RCS, Chin. Phys. C 37, 067001 (2013).

[5] I. Sakai et al., H-painting injection system for the J-PARC $3-\mathrm{GeV}$ high intensity proton synchrotron, in Proceedings of the 20th Particle Accelerator Conference, Portland, OR, 2003 (IEEE, New York, 2003), pp. 1512-1514.

[6] J. Beebe-Wang, Y. Y. Lee, D. Raparia, and J. Wei, Transverse phase space painting for SNS accumulator ring injection, in Proceedings of the 18th Particle Accelerator Conference, New York, 1999 (IEEE, New York, 1999), pp. $1743-1745$.

[7] J. D. Gabambos, J. A. Holmes, and D. K. Olsen, ORBIT user's manual, SNS/ORNL/AP Technical Note No. 011.1999.

[8] P. K. Saha, Y. Shobuda, H. Hotchi, N. Hayashi, T. Takayanagi, H. Harada, and Y. Irie, Direct observation of the phase space footprint of a painting injection in the Rapid Cycling Synchrotron at the Japan Proton Accelerator Research Complex, Phys. Rev. ST Accel. Beams 12, 040403 (2009).

[9] J. Safranek, G. Portmann, and A. Terebilo, Matlab-based Loco, in Proceedings of the 8th European Particle Accelerator Conference, Paris, 2002 (EPS-IGA and CERN, Geneva, 2002), pp. 1184-1186.

[10] A. Terebilo, Accelerator Toolbox for Matlab, SLACPUB8732, 2001, http://www.slac.stanford.edu/grp/ssrl/ spear/at/.

[11] C. Gardener and L. Ahrens, A method for determining the position, angle and other injection parameters of a short pulsed beam in the brookhaven AGS, in Proceedings of the 11th Particle Accelerator Conference, PAC-1985, Vancouver, BC, Canada, 1985 (IEEE, New York, 1985), pp. $1888-1890$.

[12] L.F. Chaparro, Signals and Systems Using MATLAB (Elsevier, New York, 2011), pp. 317-318. 